\title{
Periodontal disease as a risk factor for sporadic colorectal cancer: results from COLDENT study
}

\author{
Amal Idrissi Janati ${ }^{1} \cdot$ Igor Karp $^{2}$. Jean-François Latulippe ${ }^{3} \cdot$ Patrick Charlebois $^{4} \cdot$ Elham Emami $^{5}$
}

Received: 2 July 2021 / Accepted: 14 December 2021 / Published online: 26 January 2022

(c) The Author(s) 2021

\begin{abstract}
Colorectal cancer remains the top leading cancer worldwide. Accumulating evidence suggests periodontal pathogens are involved in colorectal carcinogenesis, indicating the need for high-quality epidemiological evidence linking periodontal disease (PD) and colorectal cancer (CRC). Thus, we conducted the first population-based case-control study that was specifically designed to investigate the association between compromised oral health and sporadic CRC. A total of 348 incident cases of colon or rectal cancer, and 310 age and sex frequency-matched controls, from the Montreal island and Laval population participated in the study. Data were collected on PD and on several CRC risk factors using validated questionnaires. A lifecourse approach was used to document long-term history regarding lifestyle factors. Multivariable unconditional logistic regression analysis was used to estimate the rate ratio (RR) quantifying the association between CRC and PD. Results showed that the rate of new diagnosis of CRC in persons with a positive history of PD was 1.45 times higher than in those with a negative history of PD adjusting for age, sex, BMI, education, income, diabetes, family history of CRC, regular use of nonsteroidal anti-inflammatory drugs, lifetime cumulative smoking, lifetime consumption of red meats, processed meats, and alcoholic drinks, and lifetime total physical activity score (adjusted $\mathrm{RR}=1.45 ; 95 \% \mathrm{CI} 1.04-2.01 ; p=0.026$ ). Our results support the hypothesis of an association between PD and sporadic CRC risk.
\end{abstract}

Keywords Periodontal disease $\cdot$ Periodontitis $\cdot$ Gingivitis $\cdot$ Colorectal cancer

$\begin{array}{ll}\text { Abbreviations } \\ \text { BMI } & \begin{array}{l}\text { Body mass index } \\ \text { CDC-AAP }\end{array} \\ & \begin{array}{l}\text { Centers for Disease Control and Pre- } \\ \text { vention and the American Academy of }\end{array} \\ \text { CI } & \begin{array}{l}\text { Periodontology } \\ \text { Confidence interval } \\ \text { CRC }\end{array} \\ \end{array}$

Elham Emami

elham.emami@mcgill.ca

1 Faculty of Dentistry, Université de Montréal, Montreal, QC, Canada

2 Department of Epidemiology and Biostatistics, Schulich School of Medicine and Dentistry, University of Western Ontario, London, ON, Canada

3 Department of Surgery, Hospital Maisonneuve-Rosemont, Université de Montréal, Montreal, QC, Canada

4 Department of Surgery, McGill University Health Centre, Montreal, QC, Canada

5 Faculty of Dental Medicine and Oral Health Sciences, McGill University, 2001 McGill College Avenue, Suite 500, Montreal, QC H3A 1G1, Canada

\begin{tabular}{|c|c|}
\hline FFQ & Food Frequency Questionnaire \\
\hline LTPAQ & Lifetime Total Physical Activity \\
\hline & Questionnaire \\
\hline MET & Metabolic-equivalent of task \\
\hline NA-NSAIDs & $\begin{array}{l}\text { Non-aspirin non-steroidal anti-inflamma- } \\
\text { tory drugs }\end{array}$ \\
\hline NSAIDs & Non-steroidal anti-inflammatory drugs \\
\hline PD & Periodontal disease \\
\hline RR & Rate ratio \\
\hline $\mathrm{RRa}$ & Adjusted rate ratio \\
\hline
\end{tabular}

\section{Background}

Colorectal cancer (CRC) is the third most commonly diagnosed cancer and the second cause of death from cancer worldwide, with over 1,900,000 new cases and over 900,000 deaths in 2020 (Global Cancer ObservatoryWorld Health Organization) [1]. While CRC can develop in inflammatory bowel diseases or hereditary syndromes, most CRC cases are sporadic [2]. Many factors are associated with sporadic CRC, including higher age, male sex, 
low socioeconomic status, diabetes mellitus, high adiposity, history of CRC in first-degree relatives, tobacco smoking, high consumption of red and processed meat, and heavy intake of alcohol [3-9]. On the other hand, physical activity and use of non-steroidal anti-inflammatory drugs (NSAIDs) decrease the risk of CRC [10-12].

In recent years, studies have suggested that periodontal disease (PD) may increase the risk of CRC. PD is a chronic inflammatory disease, caused by dysbiosis in plaque biofilms, and characterized by progressive destruction of the tooth-supporting tissues [13]. PDs include gingivitis, which refers to gingival inflammation caused by bacteria accumulating in the gingival margin, and periodontitis, where the breakdown of teeth-supporting tissues occurs [14]. The disease manifests in gingival bleeding, clinical attachment loss and radiographically assessed alveolar bone loss, and presence of periodontal pockets. Severity and extent of clinical manifestations increase with disease progression and can reach a high level of tooth mobility and, ultimately, tooth loss in advanced stages [13]. Beyond oral health, PD seems to also impact some extra-oral health outcomes and has been found to be associated with major systemic diseases, including cardiovascular, respiratory, chronic kidney, and metabolic diseases, adverse pregnancy outcomes, rheumatoid arthritis, and cancers [15]. The putative mechanism of PD and cancer association involves the spread of periodontal pathogens to extra-oral sites, dissemination of bacteria endotoxins, and release of inflammation products directly into the bloodstream. Chronic inflammation, on the other hand, promotes carcinogenesis by induction of gene mutations, inhibition of apoptosis, stimulation of angiogenesis, cell proliferation, and epigenetic alterations $[16,17]$. PD has been linked to gastrointestinal cancers, but the strength of evidence differs across cancer sites [18]. To date, several papers have been published on the association of CRC and PD [19-27], all of which relied on secondary analysis of data from studies that had been designed for other purposes. These papers suffer from various methodological limitations including, notably, misclassification of PD status and other relevant characteristics, residual confounding [28], and the low number of documented CRC cases. As a result, the etiologic role of PD in the occurrence of CRC remains uncertain. Thus, to better assess the possible etiologic role of PD in the occurrence of CRC, valid and reproducible epidemiological evidence is needed.

\section{Objective}

The objective of this study was to investigate whether PD increases the risk of sporadic CRC.

\section{Methods}

\section{Study design}

COLDENT study is a "population-based" case-control study that was carried out in the Montreal metropolitan area (Montreal island and Laval), Quebec, from January 2013 to December 2019. The CRC cases were instances of histologically confirmed colon or rectal cancer diagnosed in the 6 months preceding their identification. CRC case identification relied on the assistance of medical staff in surgery and oncology departments of five main hospitals providing CRC care to residents of Montreal island and Laval. The control series was selected by random sampling of age- and sex-based strata of the population of Montreal island and Laval by relying on the Quebec Electoral Office lists during 2013-2019. Specifically, a control-to-case ratio of approximately 1:1 was aimed at across the strata defined by age (within 10 year categories) and sex. The identified/selected subjects were sent the study introductory letter and received a phone call from research staff in the following week. Several callbacks were made for nonresponding numbers. The inclusion criteria for both cases and controls were as follows: (1) aged 40-80 years old; (2) resident of montreal island or laval; (3) Canadian citizen; (4) speak English and/or French; (5) no prior diagnosis of cancer; and (6) no prior diagnosis of an inflammatory or a hereditary bowel disease, including lynch syndrome, hereditary non-polyposis colorectal cancer, familial adenomatous polyposis, and related polyposis. Eligible respondents who agreed to participate in the study were invited to complete a multi-item study questionnaire in either a face-to-face or phone interview. If an eligible responder was unable to attend the interview, the questionnaire was offered for self-administration. In that case, the participant was instructed on questionnaire completion and was called back by research staff upon receipt of the completed questionnaire. Face-to-face interviews were carried out in research units, at the participant's home, or in hospitals for CRC patients.

The study was approved by the Research Ethics Committees of all participating institutions, and all study participants were given all the information needed before they signed the study consent form.

\section{Data collection}

Data on PD were collected using eight validated questions that address three categories of information on the subject's periodontal condition: history of diagnosis or treatment of PD; symptoms or complications of PD; and 
Table 1 Questions on self-reported periodontal disease

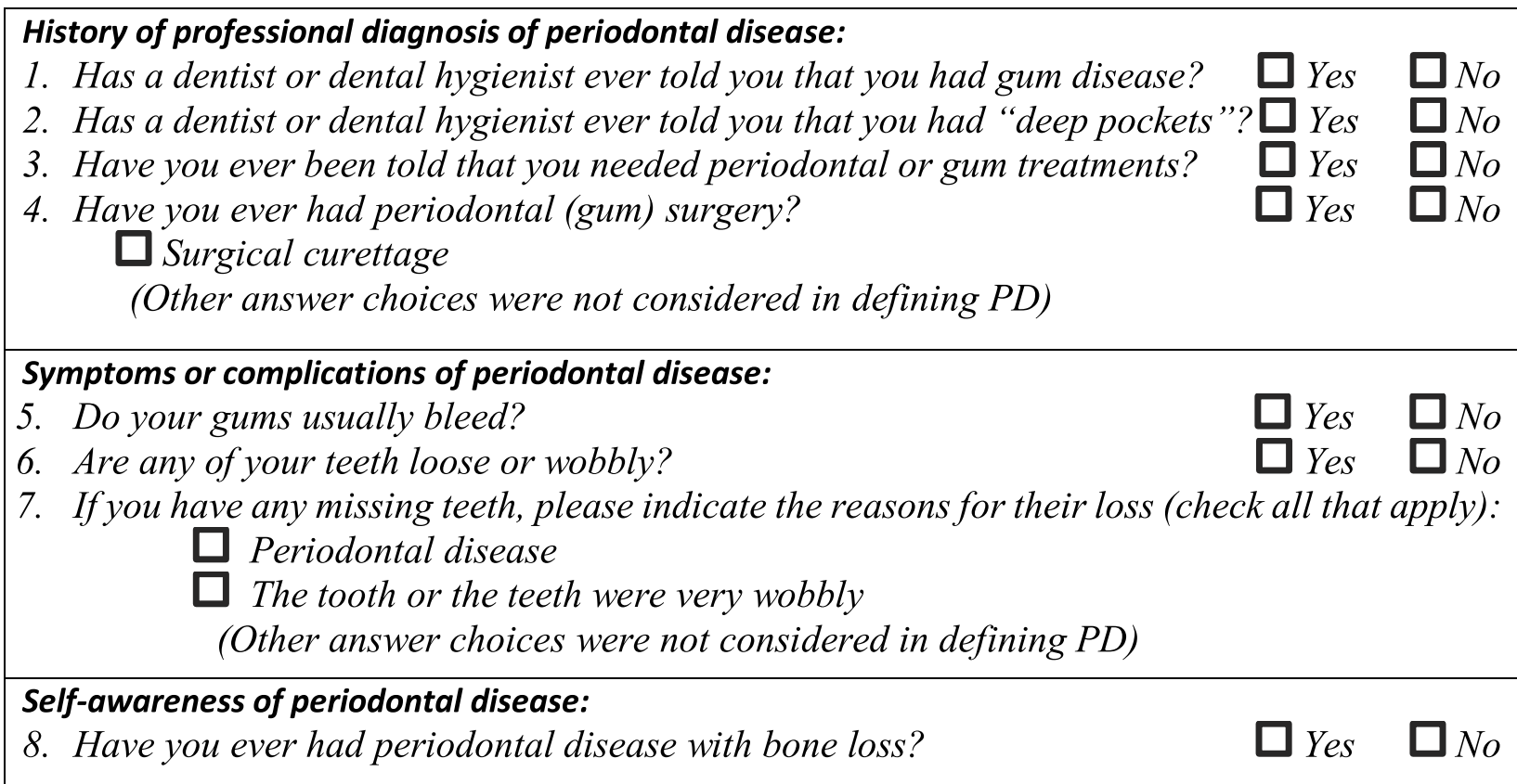

self-awareness of PD [29, 30] (see Table 1). For each category of information, questions with the highest diagnostic performance indicators were selected, based on results of a previous systematic review by Blicher [30] on validity of self-reported periodontal disease measurements. In a more recent systematic review with meta-analysis, by Abbood [29], on self-reported PD, the estimated pooled diagnostic odds ratios $(95 \% \mathrm{CI})$ of moderate PD by the questions on previous treatment of PD (deep cleaning); tooth mobility without injury; gum bleeding; and self-awareness of having gum disease were 2.38 (1.35-4.2), 6.99 (3.17-15.43), $1.40(0.91-2.16)$, and $3.20(2.23-4.57)$, respectively, and 11.72 (4.12-33.36), 2.24 (1.05-4.80), 1.95 (1.25-3.03), and 3.35 (2.17-5.18), respectively, for severe PD [29]. Moderate and severe PD were defined based on the gold standard PD case definition by the Centers for Disease Control and Prevention and the American Academy of Periodontology (CDC-AAP) [31].

Further, seven study-questionnaires were administered: Sociodemographic and Medical History, Smoking, Height and Weight, Anti-inflammatory Medications, Oral Health, Food Frequency (FFQ), and Lifetime Total Physical Activity (LTPAQ) questionnaires [32-36]. Through administration of these questionnaires, we collected data on history of cigarette smoking, including age started, age ended, years quit during period of usage, and intensity (number of cigarettes smoked per day, per week, or per month). Participants were asked to report all occupational, household, and recreational activities they had done in their lifetime. The minimum threshold for an activity to be reported in the LTPAQ is
$124 \mathrm{~h} /$ year for occupational, $112 \mathrm{~h} /$ year for household, and 32 weeks/year for recreational activities [37]. Each activity was described in terms of duration (age started and age ended), frequency (number of hours per week, weeks per month, and months per year of activity practice), and intensity: light, moderate, and vigorous. Weak intensity was only used for occupational activities to describe those that require sitting with minimal walking.

For dietary risk factors, data were collected on intake of different kinds of red meats and processed meats and of different alcoholic beverages since adulthood. Specifically, red meats referred to meat in hamburgers, beef, pork, and lamb; processed meats referred to bacon, hot dogs, or other kinds of processed meats as salami, bologna, and sausages; and alcoholic drinks referred to beer, wine, and liquor. The FFQ was then administered for four age periods: $20-34,35-49$, $50-64$, and $65-80$ years. Interviewers relied on the lifetime grid technique to enhance recall accuracy [38].

\section{Coding of data on periodontal disease and covariates}

Subjects were classified as having a positive history of PD if they reported previous professional diagnosis or treatment of $\mathrm{PD}$, and/or if they had experienced either frequent gingival bleeding, or tooth loss caused by tooth mobility or PD, and/ or if they were self-aware of having PD. Periodontal health status of participants who answered Yes to only question 6 about tooth mobility (see questions in Table 1) was considered as unknown since tooth mobility could also be caused 
by an occlusal trauma in a healthy periodontium. Participants were considered as "unexposed" to PD if none of the responses they provided to the 8 questions was positive.

Covariates for adjustment included age, gender, education attainment (elementary school vs. high school and $\geq$ college), annual personal income, body mass index (BMI), history of type II diabetes, history of CRC in first-degree relatives, history of regular use of NSAIDs (Yes/No), lifetime measure of cigarette smoking (as quantified by packsyears), lifetime measures of consumption of red meats, processed meats, and alcohol, as well as lifetime cumulative physical activity score.

Regular use of NSAIDs was defined as use of at least one tablet/capsule of NSAIDs per month for six continuous months or longer, of aspirin and non-aspirin NSAIDs (NANSAIDs). The number of packs-years was calculated as the product of the number of cigarettes smoked per day divided by 20 and the number of years smoked. Lifetime measures of consumption of red meats, processed meats, and alcoholic drinks were calculated as the average number of consumed weekly servings for red and processed meats, and of daily drinks for alcohol, since the participant was 20 years old.

Lifetime physical activity score was represented by the average amount of total energy expended during occupational, recreational, and household activities, expressed by metabolic-equivalent of task (MET) in MET-hour/week/year [37, 39-41]. For this, we assigned MET values to the intensity of physical activities: weak as 1.5 , light as 2.5 , moderate as 4 , and vigorous as 8 . To consider both the effects of duration and intensity, each activity was converted into energy expended by multiplying its assigned MET value with the reported hours spent in the activity per year, and the number of years the activity lasted. All subject activities were then summed to derive subject lifetime cumulative energy expended in MET-hours-years. This cumulative measure was then divided by the individual's age (in years) and by 52 (i.e., the number of weeks in a year) to derive physical activity scores in MET-hour/week/year.

\section{Statistical analysis}

The distributions of potential confounders in the case and control series were examined by calculating the median and inter-quartile range for continuous variables, and percentage for categorical variables.

We fitted multivariable unconditional logistic regression models to estimate the rate ratio (RR) quantifying the association between CRC and PD. Specifically, in the multivariable models, the RR was adjusted for the matching variables (age and sex) and for all the other potential confounders, namely, education attainment, annual personal income, BMI, history of type II diabetes, history of CRC in first-degree relatives, history of regular use of aspirin and NA-NSAIDs, lifetime measure of cigarette smoking, lifetime measure of consumption of red meats, lifetime measure of consumption of processed meats, lifetime measure of consumption of alcohol, and lifetime cumulative physical activity score. The linearity in the logit was assessed for all continuous independent variables, namely age, BMI, annual personal income, lifetime measure of consumption of red meats, processed meats, and alcoholic drinks, and lifetime physical activity score, using the Box-Tidwell test, which involves adding simultaneously interaction terms of each continuous variable and its natural logarithm (Xi multiplied by $\ln (\mathrm{Xi})$ ) to the multivariable regression model [42]. The test revealed that none of the interaction terms involving the above-mentioned continuous covariates was statistically significant at the alpha level of 0.05 .

The percentages for missing data for each variable were less than $10 \%$, except for regular use of aspirin (23\%), which was absent from the NSAID questionnaire during the first year of the study (see Supplementary Table S1). Missing data were addressed with the multiple imputation method using the Expectation-Maximization with Bootstrapping algorithm [43]: 10 complete datasets were generated to produce pooled "final" RR estimates along with their corresponding $95 \%$ confidence intervals (CIs). To improve performance of the imputation algorithm, auxiliary variables were included for imputation, in addition to all the variables included in the "associational" models. Continuous variables with asymmetric distribution, or extreme values, as for cumulative cigarette smoking, lifetime measures of consumption of red meats, processed meats, and alcoholic drinks, were log-transformed $(\log 10(\mathrm{Xi})$ or $\log 10(\mathrm{Xi}+1))$ before imputation. Imputed values of continuous variables were restricted to the observed minimum and maximum values. Imputation was done respecting the scale of each variable (i.e., continuous, ordinal, or categorical) with the Amelia II package in R, version 3.5.3 [44].

\section{Results}

A total of 1040 potentially eligible cases of CRC were identified from the five participating hospitals. We approached 972 patients, of whom 483 did not meet the study eligibility criteria, mainly because of their residence area $(n=109)$, previous diagnosis of cancer $(n=105)$, long time since CRC diagnosis $(n=93)$, age $(n=89)$, and language criteria $(n=56)$. Twenty-nine were not able to participate (16 were too ill or too busy because of their cancer treatments, eight had a cognitive or mental illness, five died between the time of their identification and the time when we attempted to reach them or meet for an interview), and 112 refused to participate. Thus, 348 CRC patients took part in the study. During the same period, 1346 CRC-free controls were sent 
the introduction letter. We were unable to reach 450 subjects, including 119 subjects whose contact details (address and/or phone number) had changed, and five subjects who had died between the time of their selection and the time when we attempted to reach them. Among 896 subjects who were reached, 186 did not meet the study eligibility criteria (mostly because of a previous cancer $(n=79)$, and not speaking French or English $(n=52)$ ), five subjects were unable to participate because of a cognitive condition, and 395 refused to participate. Thus, 310 control subjects took part in the study (see flow chart in Fig. 1).

Table 2 presents the distributions of the CRC cases and controls (both overall and according to their PD-history status) according to sociodemographic and other relevant characteristics. Regarding sociodemographic and anthropometric characteristics, cases and controls showed similar mean age (63 years old) and BMI $\left(26 \mathrm{~kg} / \mathrm{m}^{2}\right)$, and similar relative frequencies of lifestyle and family history of CRC in firstdegree relatives. However, the case series showed higher relative frequencies of male gender, elementary and high school education, and history of type II diabetes. Further, the median personal income in the cases was lower than in the controls. The relative frequency of regular use of aspirin was higher in the cases than in the controls, while the relative frequency of regular use of NA-NSAIDs was lower in the cases than in the controls. Considering assessed behavioral CRC risk factors in lifetime, the cases showed a higher proportion of smokers, higher average number of packs-years, higher level of intake of processed meats, and higher average MET-hour/week/year scores in their occupational activities.

The prevalence of $\mathrm{PD}$, including gingivitis and periodontitis, was higher in the case group (46\%) than in the control group (38\%). Overall, cases were more likely to report previous diagnosis or a symptom of $\mathrm{PD}$, or to be aware of having PD with bone loss, than controls. The complication of advanced PD, tooth loss due to mobility or PD, was less frequent ( $8 \%$ in cases and $4.8 \%$ in controls).

Results from multiple logistic regression analysis showed that the rate of (new diagnosis of) CRC in persons with a positive history of PD was 1.4 times higher than in those with a negative history of PD when adjusting only for age and gender (adjusted rate ratio $\left(\mathrm{RR}_{\mathrm{a}}=1.4 ; 95 \% \mathrm{CI}\right.$ $1.02-1.91 ; p=0.037)$ and 1.45 times higher than in those with a negative history of PD when adjusting, in addition, for BMI, education, income, diabetes, family history of CRC, regular use of aspirin and NA-NSAIDs, lifetime cumulative smoking, lifetime consumption of red meats, processed meats, and alcoholic drinks, and lifetime total physical activity score $\left(\mathrm{RR}_{\mathrm{a}}=1.45 ; 95 \%\right.$ CI $\left.1.04-2.01 ; p=0.026\right)$. The results are summarized in Table 3.
Fig. 1 Flow chart of COLDENT study recruitment

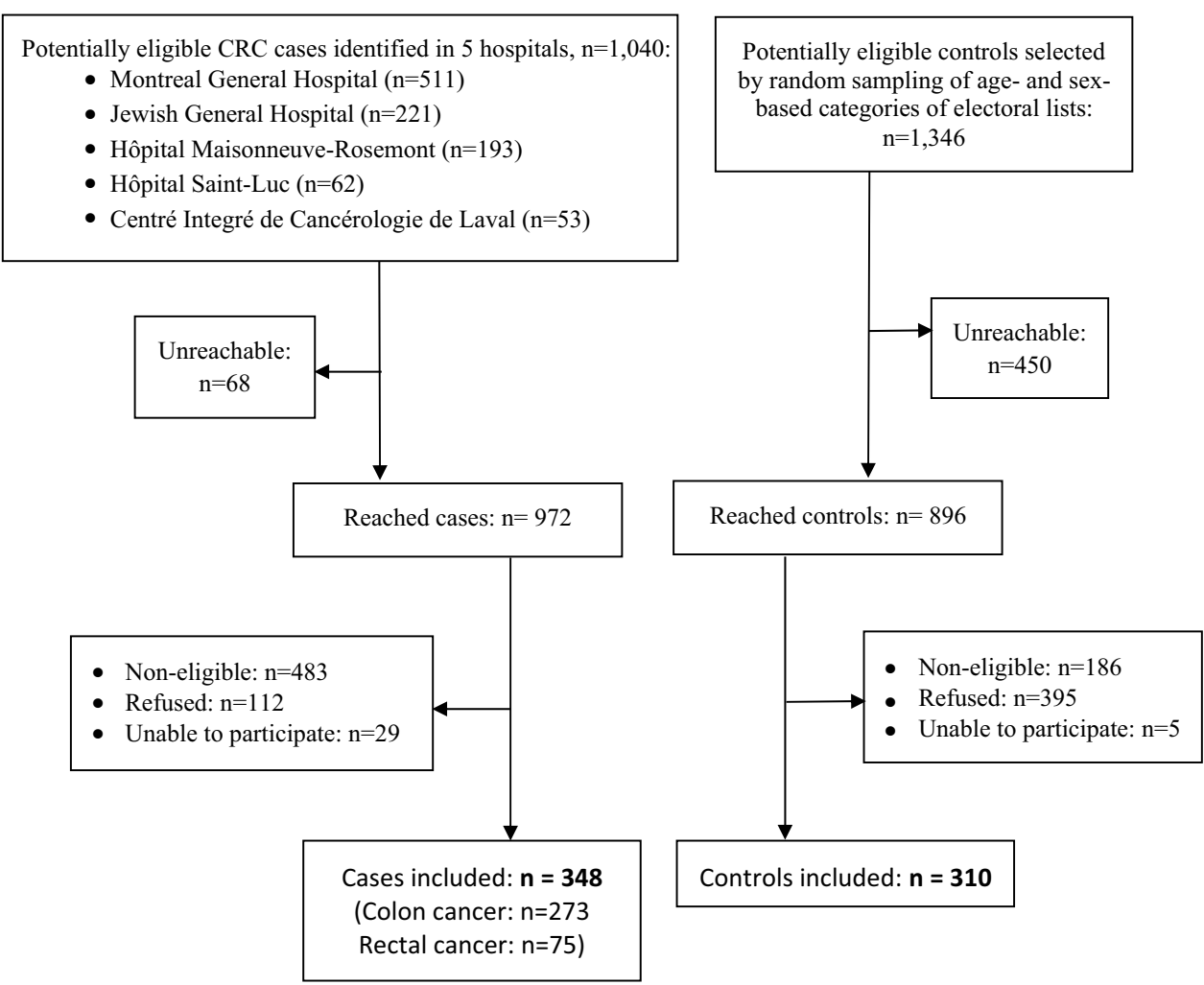


Table 2 Sociodemographic characteristics and CRC risk factors in COLDENT study participants

\begin{tabular}{|c|c|c|c|c|}
\hline \multirow[t]{2}{*}{ Characteristic } & \multirow{2}{*}{$\begin{array}{l}\text { Cases, } n=348 \\
n(\%) \\
\text { Total }\end{array}$} & \multicolumn{3}{|l|}{$\begin{array}{l}\text { Controls, } n=310 \\
n(\%)\end{array}$} \\
\hline & & Total & $\begin{array}{l}\text { Positive history of PD } \\
(n=117)\end{array}$ & $\begin{array}{l}\text { Negative history of PD } \\
(n=183)\end{array}$ \\
\hline Age, years (mean (SD)) & $63.2(9.8)$ & $63.1(9.8)$ & $61.4(9.6)$ & $63.7(9.8)$ \\
\hline \multicolumn{5}{|l|}{ Gender } \\
\hline Male & $215(62)$ & $170(55)$ & $66(56)$ & $98(54)$ \\
\hline \multicolumn{5}{|l|}{ Canadian born } \\
\hline Yes & $216(62)$ & $210(68)$ & $77(66)$ & $126(69)$ \\
\hline \multicolumn{5}{|l|}{ Native tongue } \\
\hline French & $155(46)$ & $183(59)$ & $63(55)$ & $112(64)$ \\
\hline English & $60(18)$ & $40(13)$ & $20(17)$ & $20(12)$ \\
\hline Other language & $123(36)$ & $75(24)$ & $32(28)$ & $42(24)$ \\
\hline \multicolumn{5}{|l|}{ Education attainment } \\
\hline Elementary school & $29(9)$ & $9(3)$ & $4(3)$ & $5(3)$ \\
\hline High school & $116(34)$ & $92(30)$ & $36(31)$ & $53(30)$ \\
\hline College or University & $197(58)$ & 199 (64) & $76(65)$ & 117 (67) \\
\hline \multicolumn{5}{|l|}{ Living alone } \\
\hline Yes & $71(21)$ & $80(26)$ & $27(23)$ & $47(26)$ \\
\hline BMI, kg /m² (mean (SD)) & $26.1(5.7)$ & $26.3(6.2)$ & $27(5.7)$ & $27.4(4.7)$ \\
\hline \multicolumn{5}{|l|}{ Family history of CRC } \\
\hline Positive & $43(12)$ & $38(12)$ & $13(11)$ & $24(13)$ \\
\hline \multicolumn{5}{|l|}{ History of diabetes } \\
\hline Positive & $69(20)$ & $39(13)$ & $14(12)$ & $23(13)$ \\
\hline \multicolumn{5}{|l|}{ History of regular use of NA-NSAIDs } \\
\hline Positive & $62(18)$ & $67(22)$ & $28(25)$ & $39(21)$ \\
\hline \multicolumn{5}{|l|}{ History of regular use of aspirin } \\
\hline Positive & $85(27)$ & $50(26)$ & $16(21)$ & $33(30)$ \\
\hline Personal income (CAD\$ per year) & $35000(50000)$ & $45000(40000)$ & $45000(50000)$ & $45000(40000)$ \\
\hline \multicolumn{5}{|l|}{ History of cigarette smoking } \\
\hline Positive & $206(59)$ & $174(56)$ & $73(62)$ & $97(53)$ \\
\hline Cigarette smoking, packs-years (Median (IQR)) & $3.6(24)$ & $2(22)$ & $2.2(17.5)$ & $1.8(23.5)$ \\
\hline Lifetime average daily alcoholic drinks ${ }^{\mathrm{a}}$ (Median (IQR)) & $0.4(1.1)$ & $0.5(1)$ & $0.4(0.9)$ & $0.5(1)$ \\
\hline \multicolumn{5}{|l|}{ Lifetime average weekly servings ${ }^{\mathrm{b}}$ of: } \\
\hline Red meats (Median (IQR)) & $4.3(3.5)$ & $4(3.5)$ & $4.4(3.5)$ & $3.9(3.3)$ \\
\hline Processed meats (Median (IQR)) & $2.6(3.5)$ & $1.8(3.3)$ & $1.8(3.2)$ & $1.8(3.3)$ \\
\hline \multicolumn{5}{|l|}{ Lifetime average MET hour/week/year (Median (IQR)) } \\
\hline Occupational & $60.3(51.6)$ & $48.7(40.8)$ & $50.5(41.5)$ & $46.9(36.5)$ \\
\hline Household & $13.4(27.6)$ & $13.1(25.1)$ & $12.3(26)$ & $13.6(23.7)$ \\
\hline Recreational & $8(14)$ & $7.7(12.8)$ & $8.4(12.7)$ & $6.5(11.3)$ \\
\hline Total physical activity & $95.7(78.2)$ & $85(53)$ & $84.8(44.9)$ & $84.9(53.6)$ \\
\hline
\end{tabular}

The frequencies presented in the table correspond to valid percentages

$C R C$ colorectal cancer, BMI body mass index, NA-NSAIDs non-aspirin non-steroidal anti-inflammatory drugs, CAD\$ Canadian dollar, $M E T$ metabolic equivalent of task, $S D$ standard deviation, $I Q R$ interquartile range

${ }^{a}$ One drink include beer $(355 \mathrm{ml}$ bottle or can $)$, wine $(180 \mathrm{ml})$, or liquor $(150 \mathrm{ml})$

${ }^{\mathrm{b}}$ One serving of red meats $=180-240 \mathrm{~g}$, one serving of processed meats $=55 \mathrm{~g}$ 
Table 3 Rate ratio (RR) for association between periodontal disease and colorectal cancer

\begin{tabular}{ll}
\hline & $\mathrm{RR}(95 \% \mathrm{CI}) ; p$ value \\
\hline Adjusted for age and sex & $1.4(1.02-1.91) ; p=0.037$ \\
Adjusted for all covariates $^{\mathrm{a}}$ & $1.45(1.04-2.01) ; p=0.026$ \\
\hline
\end{tabular}

${ }^{a}$ Age, sex, education attainment, annual personal income, BMI, history of type II diabetes, history of CRC in first-degree relatives, history of regular use of aspirin and non-aspirin non-steroidal antiinflammatory drugs, lifetime measure of cigarette smoking, lifetime measure of consumption of red meats, lifetime measure of consumption of processed meats, lifetime measure of consumption of alcohol, and lifetime cumulative physical activity score

\section{Discussion}

In this case-control study, we investigated the association between PD and CRC, and relied on multiple self-reported measurements to ascertain the PD status. Our findings suggest that the $\mathrm{CRC}$ rate is increased in persons with a positive history of PD compared with persons with a negative history of PD, even upon adjustment for a number of potential confounders. Studies that previously examined the association between CRC and self-reported PD [22, 23, 25, 26] assessed PD status with only one question, which was about previous professional diagnosis of $\mathrm{PD}$, among a cohort of American male health professionals and a cohort of elderly women [23, 26]; self-awareness of periodontal bone loss in American women nurses [22]; or a clinical sign of PD (tooth mobility with consequent tooth loss) in a cohort of adult Sweden twins [25]. Unlike those studies, we ascertained selfreported PD exposure using a combination of questions that concerned the three types of information together. Using only one question can be expected to result in PD-status misclassification, especially in persons with lower education or socio-economic level [45]. For example, questions related to professional diagnosis will favor accurate answers in subjects who had more access to dental care. [29, 30] Thus, persons who have not visited a dentist in years may not report any previous diagnosis of PD and will be classified as unexposed, while in fact they could well have the disease, especially given their poorer access to, and lower levels of use of, dental care. According to the Canadian Health Measures Survey 2007-2009, 48\% of Canadian adults who have not been to a dental professional in last year had gingivitis, and $48 \%$ of Canadian adults from the lower income group had gingivitis, compared with $25 \%$ of Canadians with higher incomes [46]. Questions about the most easily perceived clinical signs, such as tooth mobility, or tooth loss caused by mobility, and PD have greater informativeness in detecting PD but may result in failure to detect early stages of the disease, including gingivitis and mild periodontitis. This justifies questioning on gingival bleeding as well. Self-awareness of one's own PD condition is more informative among educated people and dental or health care users. To conclude, combining different questions - as recommended by Blicher et al. [30] and Abbood et al. [29] in their systematic reviews' conclusions-will enhance the ability of the questions to identify history of PD. Those authors also recommended using variations of the same question in order to stimulate the person's memory; thus, we used different questions to retrieve a previous diagnosis or treatment of $\mathrm{PD}$.

According to our self-reported PD definition, we found that $38 \%$ of controls had either gingivitis or periodontitis, and if we refer to those who reported tooth loss because of mobility and PD, we can consider $5 \%$ of controls had advanced PD. These statistics seem to be in line with those from the Canadian Health Measures Survey (2007-2009), where partial-mouth periodontal examination was used, and which estimated that $32 \%$ of Canadian adults (20-79 years of age) had gingivitis, $21 \%$ of adults with natural teeth had, or had had, a moderate or a severe periodontal (gum) problem, and $4 \%$ of Canadian adults had severe periodontal disease [46]. According to complete-mouth examination in the National Health and Nutrition Examination Surveys (2009-2014), and the CDC-AAP case definition [31], the estimated prevalence of total periodontitis in dentate US adults aged $30-79$ years was $42 \%$, including $8 \%$ severe periodontitis.

We found a statistically significant association of CRC with PD $\left(\mathrm{RR}_{\mathrm{a}}=1.45 ; 95 \%\right.$ CI 1.04-2.01) $(p=0.026)$, adjusting for a number of potential confounders. Similarly, two previous studies have reported a positive association of $\mathrm{CRC}$ with PD. The first is a large retrospective cohort study $(n=106,487)$ based on administrative data from Taiwan's National Health Insurance Research Database [19]. The estimated hazard ratio (HR) (95\% CI) was 1.64 (1.50-1.80), adjusting only for age, gender, and comorbidity. PD classification in that study relied on established administrative codes given to clinical diagnosis of gingivitis and periodontitis, and no additional information was provided on any standardized clinical measures or definition of PD. In addition to this, and to the high risk of residual confounding due poor adjustment, using the Insurance Health database was criticized in another report for the significant risk of misclassification due to deliberate over-coding by health and dental care providers to avoid refusal of reimbursement by medical insurance [47]. The results of this study should thus be interpreted with great caution. In the second study, Arora [25] analyzed data from a large Swedish prospective cohort study of homo- and heterozygous twins, which had been originally designed to study the role of environmental and genetic factors in cardiovascular disease and cancer. Their analysis included 15,333 twins who answered the question on periodontal status: "Have you noticed that some of your own teeth have come loose or fallen out on their 
own?" Participants were then classified as having PD if at least half of their teeth were wobbly, indicating that PD is at an advanced stage. Participants who reported having a few loose teeth were separately classified as having minor mobility. Almost 6\% of participants had advanced PD and an additional $12 \%$ of participants reported minor tooth mobility. Participants had a median age at study entry of 51 years and followed-up for a median period of 27 years, with a total of 200 CRC cases documented. Upon adjustment for gender, age, education, employment, number of siblings, smoking status ( 5 categories counting number of packs per day), smoking status of partner, alcohol status (current, former, never), body mass index (4 categories), and diabetes, the estimated HR (95\% CI) was 1.62 (1.13-2.33). Unfortunately, the list of potential confounders adjusted for did not include dietary risk factors and physical activity level. Furthermore, the PD measure in that study excluded gingivitis and earlystage periodontitis.

Different results were reported by Momen-Heravi [22], who analyzed data from the American Nurses' Health Study. The analysis included a subsample of 69,656 participants who were asked if they had a history of periodontal bone loss (in study cycle of 1998). Where the answer was yes, participants indicated the severity of the bone loss (none, mild, moderate/severe). After 18 years of follow-up, a total of 739 CRC was documented for this analysis. Overall, the study results suggested no association between history of periodontal bone loss and CRC (HR $=0.89 ; 95 \%$ CI $0.72-1.10)$, although they were weakly suggestive of positive association for moderate/severe bone loss and CRC (HR $=1.22$; $95 \%$ CI 0.91-1.63), adjusting for age, ethnicity, smoking, history of CRC in first-degree relatives, history of sigmoidoscopy/colonoscopy, current physical activity, regular aspirin use, multivitamin use, diabetes, alcohol consumption, BMI, energy-adjusted intake of total calcium, vitamin $\mathrm{D}$, folate, red meat and processed meat, and hormonal replacement therapy). The study indeed has many strengths, such as its prospective design, large sample size, and adjustment for numerous potential confounders.

Results from these epidemiological studies on the association between PD and CRC (or colorectal adenoma [48, 49]) have been synthesized in a recent meta-analysis, by Xuan [50]. Specifically, these authors reported that periodontal disease was statistically significantly associated with colorectal tumor (pooled "relative risk" and 95\% CI: 1.25 (1.06-1.38), although there was high heterogeneity across studies $\left(I_{2}=83.9 \%\right)[50]$.

The oral cavity can indeed serve as a reservoir for the systemic dissemination of pathogenic bacteria and their toxins, leading to infections and inflammations in distant bodily sites, and several oral species were identified in infections at extra-oral sites [51]. Scannapieco and Panagakos [52] suggested four potential pathways that may allow oral bacteria and gingival inflammation to influence systemic health, including bacteremia, systemic spread of locally produced inflammatory mediators, eliciting an autoimmune response, and aspiration or ingestion of oral bacteria into the intestine or respiratory tract [52].As for CRC, a periodontal pathogen, Fusobacterium nucleatum, has been particularly involved in CRC tumorigenesis, and its presence in colorectal mucosa and feces has been found to be associated with CRC [53]. The development of CRC is, on the other hand, strongly influenced by the inflammatory condition of the colon, as shown in patients with inflammatory bowel disease, where chronic and severe inflammation of the colon increases their risk of developing CRC [54].

To our knowledge, the COLDENT project was the first epidemiological study specifically designed to assess the association between PD and CRC. In the study we used rigorous methods for documentation of the main exposure, study outcome, and the potential confounding factors. Moreover, we followed a life-course approach to document longterm history regarding lifestyle factors and other relevant characteristics. We also chose to assess PD based on selfreported measures, because they allow easier standardization of the exposure measurements and definition than dental examination, which is sensitive to inter- and intra-examiner variation.

Our results support the hypothesis of an association between PD and sporadic CRC risk. Further epidemiological studies aimed at production of high-quality evidence on the putatively causal relation between PD and CRC occurrence and on the possible mechanisms underlying that relation are recommended.

Supplementary Information The online version contains supplementary material available at https://doi.org/10.1007/s10552-021-01541-y.

Acknowledgements We thank all participants in the COLDENT study, Dr Carole Richard, Dr Nathalie Aucoin, Dr Marylise Boutros, and the late Dr Phillip Gordon, as well as all nurses and administrative staff who helped in study participant identification and recruitment in the participating hospitals: Montreal General Hospital, Jewish General Hospital, Hôpital Maisonneuve-Rosemont, Hôpital Côte Saint Luc du CHUM, and Centre Intégré de Cancérologie de Laval. We also thank research assistant Mrs Nancy Faraj, and statistician Mr Miguel Chagnon who helped with multiple imputation.

Funding This project was supported by an operating grant from the Cancer Research Society. AIJ was supported by a doctoral scholarship from the Fonds de Recherche du Québec-Santé.

Data availability Data supporting the findings of this study will be available if the institutional review boards accept and upon a request from the corresponding author.

\section{Declarations}

Conflict of interest The authors declare no competing interests. 
Ethical approval The study was approved by the Research Ethics Committees of all participating institutions, and all study participants were given all the information needed before they signed the study consent form.

Consent for publication Not applicable.

Open Access This article is licensed under a Creative Commons Attribution 4.0 International License, which permits use, sharing, adaptation, distribution and reproduction in any medium or format, as long as you give appropriate credit to the original author(s) and the source, provide a link to the Creative Commons licence, and indicate if changes were made. The images or other third party material in this article are included in the article's Creative Commons licence, unless indicated otherwise in a credit line to the material. If material is not included in the article's Creative Commons licence and your intended use is not permitted by statutory regulation or exceeds the permitted use, you will need to obtain permission directly from the copyright holder. To view a copy of this licence, visit http://creativecommons.org/licenses/by/4.0/.

\section{References}

1. Sung H, Ferlay J, Siegel RL et al (2021) Global cancer statistics 2020: GLOBOCAN estimates of incidence and mortality worldwide for 36 cancers in 185 countries. CA Cancer J Clin 71(3):209-249

2. Ponz de Leon M, Percesepe A (2000) Pathogenesis of colorectal cancer. Dig Liver Dis 32(9):807-821

3. Coughlin SS (2020) Social determinants of colorectal cancer risk, stage, and survival: a systematic review. Int J Colorectal Dis 35(6):985-995

4. Deng L, Gui Z, Zhao L, Wang J, Shen L (2012) Diabetes mellitus and the incidence of colorectal cancer: an updated systematic review and meta-analysis. Dig Dis Sci 57(6):1576-1585

5. Ning Y, Wang L, Giovannucci EL (2010) A quantitative analysis of body mass index and colorectal cancer: findings from 56 observational studies. Obes Rev 11(1):19-30

6. Botteri E, Iodice S, Bagnardi V et al (2008) Smoking and colorectal cancer: a meta-analysis. JAMA 300(23):2765-2778

7. Händel MN, Rohde JF, Jacobsen R et al (2020) Processed meat intake and incidence of colorectal cancer: a systematic review and meta-analysis of prospective observational studies. Eur J Clin Nutr 74(8):1132-1148

8. Smolińska K, Paluszkiewicz P (2010) Risk of colorectal cancer in relation to frequency and total amount of red meat consumption. Systematic review and meta-analysis. Arch Med Sci 6(4):605-610

9. McNabb S, Harrison TA, Albanes D et al (2020) Meta-analysis of 16 studies of the association of alcohol with colorectal cancer. Int J Cancer 146(3):861-873

10. Robsahm TE, Aagnes B, Hjartåker A et al (2013) Body mass index, physical activity, and colorectal cancer by anatomical subsites: a systematic review and meta-analysis of cohort studies. Eur J Cancer Prev 22(6):492-505

11. Bosetti C, Santucci C, Gallus S, Martinetti M, La Vecchia C (2020) Aspirin and the risk of colorectal and other digestive tract cancers: an updated meta-analysis through 2019. Ann Oncol 31(5):558-568

12. Tomić T, Domínguez-López S, Barrios-Rodríguez R (2019) Nonaspirin non-steroidal anti-inflammatory drugs in prevention of colorectal cancer in people aged 40 or older: a systematic review and meta-analysis. Cancer Epidemiol 58:52-62
13. Papapanou PN, Sanz M, Buduneli N et al (2018) Periodontitis: Consensus report of workgroup 2 of the 2017 World Workshop on the Classification of Periodontal and Peri-Implant Diseases and Conditions. J Periodontol 89(Suppl 1):S173-S182

14. Petersen PE, Ogawa H (2012) The global burden of periodontal disease: towards integration with chronic disease prevention and control. Periodontology 2000 60(1):15-39

15. Nazir MA (2017) Prevalence of periodontal disease, its association with systemic diseases and prevention. Int J Health Sci (Qassim) 11(2):72-80

16. Castellarin M, Warren RL, Freeman JD et al (2012) Fusobacterium nucleatum infection is prevalent in human colorectal carcinoma. Genome Res 22(2):299-306

17. Kraus S, Arber N (2009) Inflammation and colorectal cancer. Curr Opin Pharmacol 9(4):405-410

18. Zhang Y, Sun C, Song EJ et al (2020) Is periodontitis a risk indicator for gastrointestinal cancers? A meta-analysis of cohort studies. J Clin Periodontol 47(2):134-147

19. Hu JM, Shen CJ, Chou YC et al (2018) Risk of colorectal cancer in patients with periodontal disease severity: a nationwide, population-based cohort study. Int J Colorectal Dis 33(3):349-352

20. Mai X, LaMonte MJ, Hovey KM et al (2016) Periodontal disease severity and cancer risk in postmenopausal women: the Buffalo OsteoPerio Study. Cancer Causes Control 27(2):217-228

21. Michaud DS, Lu J, Peacock-Villada AY et al (2018) Periodontal disease assessed using clinical dental measurements and cancer risk in the ARIC Study. J Natl Cancer Inst 110(8):843-854

22. Momen-Heravi F, Babic A, Tworoger SS et al (2017) Periodontal disease, tooth loss and colorectal cancer risk: results from the Nurses' Health Study. Int J Cancer 140(3):646-652

23. Nwizu NN, Marshall JR, Moysich K et al (2017) Periodontal disease and incident cancer risk among postmenopausal women: results from the Women's Health Initiative Observational Cohort. Cancer Epidemiol Biomarkers Prev 26(8):1255-1265

24. Ahn J, Segers S, Hayes RB (2012) Periodontal disease, Porphyromonas gingivalis serum antibody levels and orodigestive cancer mortality. Carcinogenesis 33(5):1055-1058

25. Arora M, Weuve J, Fall K, Pedersen NL, Mucci LA (2010) An exploration of shared genetic risk factors between periodontal disease and cancers: a prospective co-twin study. Am J Epidemiol 171(2):253-259

26. Michaud DS, Liu Y, Meyer M, Giovannucci E, Joshipura K (2008) Periodontal disease, tooth loss, and cancer risk in male health professionals: a prospective cohort study. Lancet Oncol 9(6):550-558

27. Hujoel PP, Drangsholt M, Spiekerman C, Weiss NS (2003) An exploration of the periodontitis-cancer association. Ann Epidemiol 13(5):312-316

28. Becher H (1992) The concept of residual confounding in regression models and some applications. Stat Med 11(13):1747-1758

29. Abbood HM, Hinz J, Cherukara G, Macfarlane TV (2016) Validity of self-reported periodontal disease: a systematic review and meta-analysis. J Periodontol 87(12):1474-1483

30. Blicher B, Joshipura K, Eke P (2005) Validation of selfreported periodontal disease: a systematic review. J Dent Res 84(10):881-890

31. Eke PI, Borgnakke WS, Genco RJ (2020) Recent epidemiologic trends in periodontitis in the USA. Periodontology 2000 82(1):257-67

32. Friedenreich CM, Courneya KS, Bryant HE (1998) The lifetime total physical activity questionnaire: development and reliability. Med Sci Sports Exerc 30(2):266-274

33. Willett WC, Reynolds RD, Cottrell-Hoehner S, Sampson L, Browne ML (1987) Validation of a semi-quantitative food frequency questionnaire: comparison with a 1-year diet record. J Am Diet Assoc 87(1):43-47 
34. Cade J, Thompson R, Burley V, Warm D (2002) Development, validation and utilisation of food-frequency questionnaires-a review. Public Health Nutr 5(4):567-587

35. Siemiatycki J, Krewski D, Franco E, Kaiserman M (1995) Associations between cigarette smoking and each of 21 types of cancer: a multi-site case-control study. Int J Epidemiol 24(3):504-514

36. Idrissi Janati A (2014) La santé orale compromise comme facteur de risque potentiel pour le cancer colorectal sporadique (COLDENT): une étude de faisabilité. Thèses et mémoires électroniques de l'Université de Montréal. http://hdl.handle.net/1866/11339. Accessed 1 June 2021

37. MacLaughlin S, Friedenreich C (2016) Cancer epidemiology and prevention research, Alberta Health Services. Lifetime Total Physical Activity Questionnaire comprehensive users' guide. https:// www.cepr.ca/questionnaires/life-time-physical-activity-questionna ire-ltpaq/. Accessed 3 January 2020

38. Berney LR, Blane DB (1997) Collecting retrospective data: accuracy of recall after 50 years judged against historical records. Soc Sci Med 45(10):1519-1525

39. Tudor-Locke C, Ainsworth BE, Washington TL, Troiano R (2011) Assigning metabolic equivalent values to the 2002 census occupational classification system. J Phys Act Health 8(4):581-586

40. Mansoubi M, Pearson N, Clemes SA et al (2015) Energy expenditure during common sitting and standing tasks: examining the 1.5 MET definition of sedentary behaviour. BMC Public Health $15: 1-8$

41. Shih IF, Liew Z, Krause N, Ritz B (2016) Lifetime occupational and leisure time physical activity and risk of Parkinson's disease. Parkinsonism Relat Disord 28:112-117

42. Menard S (2002) Applied logistic regression analysis, 2nd edn. Sage Publications, Inc, USA. https://doi.org/10.4135/9781412983 433

43. Takahashi M (2017) Statistical inference in missing data by mcmc and non-mcmc multiple imputation algorithms: assessing the effects of between-imputation iterations. Data Sci J 16(37):1-17. https://doi.org/10.5334/dsj-2017-037

44. Honaker J, King G, Blackwell M (2011) Amelia II: a program for missing data. J Statistical Softw 45(7). http://www.jstatsoft.org/ Accessed 20 Dec 2020
45. Tomar S (2005) Individual self-reported items are not accurate markers for a history of chronic periodontitis. J Evid Based Dent Pract 5(4):219-221

46. Canada H (2010) Summary report on the findings of the oral health component of the Canadian Health Measures Survey 2007-2009

47. Hsieh CY, Su CC, Shao SC et al (2019) Taiwan's National Health Insurance Research Database: past and future. Clin Epidemiol 11:349-358

48. Kim GW, Kim YS, Lee SH et al (2019) Periodontitis is associated with an increased risk for proximal colorectal neoplasms. Sci Rep $9(1): 7528$

49. Lee D, Jung KU, Kim HO, Kim H, Chun HK (2018) Association between oral health and colorectal adenoma in a screening population. Medicine (Baltimore) 97(37):e12244

50. Xuan K, Jha AR, Zhao T, Uy JP, Sun C (2020) Is periodontal disease associated with increased risk of colorectal cancer? A meta-analysis. Int J Dent Hyg. https://doi.org/10.1111/idh.12483

51. Han YW, Wang X (2013) Mobile microbiome: oral bacteria in extra-oral infections and inflammation. J Dent Res 92(6):485-491

52. Scannapieco FA, Panagakos FS (2011) Periodontal inflammation: from gingivitis to systemic disease? In: InTech (ed) Gingival diseases: their aetiology, prevention and treatment. Croatia, pp 149-172

53. Idrissi Janati A, Karp I, Laprise C, Sabri H, Emami E (2020) Detection of Fusobaterium nucleatum in feces and colorectal mucosa as a risk factor for colorectal cancer: a systematic review and meta-analysis. Syst Rev 9(1):276

54. Brennan CA, Garrett WS (2016) Gut microbiota, inflammation, and colorectal cancer. Annu Rev Microbiol 70:395-411

Publisher's Note Springer Nature remains neutral with regard to jurisdictional claims in published maps and institutional affiliations. 